\title{
MOLECULAR TARGETED TREATMENT OF METASTATIC COLORECTAL CANCER: THE CARDIOVASCULAR ADVERSE EFFECTS OF BEVACIZUMAB AND CETUXIMAB
}

\author{
ALEXANDRA GHERMAN ${ }^{1}$, CĂLIN CĂINAP ${ }^{1,2}$, \\ ANNE-MARIE CONSTANTIN ${ }^{3}$, SÎNZIANA CETEAN ${ }^{4}$, \\ SIMONA SORANA CĂINAP ${ }^{5,6}$
}

\author{
${ }^{1} 11$ th Department of Oncology, Iuliu Hatieganu University of Medicine and \\ Pharmacy, Cluj-Napoca, Romania \\ ${ }^{2}$ Prof. Dr. Ion Chiricuta Oncology Institute, Cluj-Napoca, Romania \\ ${ }^{3} 1$ st Department of Morphological Sciences, Iuliu Hatieganu University of \\ Medicine and Pharmacy, Cluj-Napoca, Romania \\ ${ }^{4}$ 2nd Department of General and Inorganic Chemistry, Faculty of Pharmacy, \\ Iuliu Hatieganu University of Medicine and Pharmacy, Cluj-Napoca, Romania \\ 52nd Pediatric Clinic, Cluj-Napoca, Romania \\ ${ }^{6} 9$ th Department of Infant Care, Iuliu Hatieganu University of Medicine and \\ Pharmacy, Cluj-Napoca, Romania
}

\begin{abstract}
Novel emerging therapies have changed paradigms in metastatic colorectal cancer. The advantages of molecular targeted treatments, either the anti-angiogenic or the anti-epidermal growth factor receptor drugs, reside in the fact that while their specificity for the cancer cell is higher, their toxicity on normal tissues is significantly lower when compared to chemotherapy. But when it comes to their safety, especially from a cardiovascular point of view, they still need to pass the test of time and further prospective studies are needed. Clinical trial patients are very well selected with regards to comorbidities and therefore, they often differ from real-life patients. In order to maximize the benefits from these drugs, we need to better identify the population at risk, understand and early diagnose their on-and off-target adverse effects and to adequately choose the diagnostic tools; with a better prevention and early treatment, the quality and quantity of our patients' lives can be significantly improved.
\end{abstract} Cetuximab

Keywords: metastatic colorectal cancer, cardiovascular toxicity, Bevacizumab,

\section{Introduction}

Colorectal cancer (CRC) represents the 2nd most frequent cancer and $13 \%$ of all human cancers. $25 \%$ of CRC patients are metastatic from the diagnosis and up to $50 \%$ present metastasis sometime during the course of the disease $[1,2]$. The therapeutic advances and multidisciplinary treatment for stage IV disease have prolonged the survival; today, the median overall survival (mOS) can reach 33 months, doubled as compared to 20

Manuscript received: 06.11.2016

Accepted: 06.12.2016

Address for correspondence: calincainap2015@gmail.com years ago $[2,3,4]$. In Romania, it is the cancer with the highest raise in incidence over the last years, currently being the most frequent digestive cancer and the 2nd most frequent cancer after the lung cancer [5].

Currently there are two major tumor pathways which are targeted by approved drugs (US Food \& Drug Administration, FDA and European Medicines Agency, EMA): angiogenic and epidermal growth factor receptor (EGFR)-mediated intracellular signaling.

One of the basic characteristics of the cancer cell and of the tumor microenvironment stromal cells is their capacity to induce angiogenesis by expressing 
pro-angiogenic factors [6]. In 1971 Folkman stated that inducing a dormant state in tumors by blocking angiogenesis improves survival of cancer patients [7]. The proangiogenic vascular endothelial growth factor (VEGF) is involved in the survival and migration of the endothelial cells and it increases the vascular permeability [8]. The most important ligand-receptor binding to activate the angiogenic pathway is between the circulating VEGF-A and VEGFR-2 (the tyrosine kinase receptor of the endothelial cell) [9]. At the moment of the "angiogenic switch" of the dormant tumor cells, the hypoxia inducible transcription factor 1 (HIF-1) induces the expression of multiple genes, generating proangiogenic proteins, VEGF included; once in the tumor microenvironment, these proteins stimulate the tumor associated macrophages to produce VEGF and other proangiogenic factors [10].

Attempts to translate these mechanisms into clinical practice have led to the discovery of drugs targeting the VEGF-VEGFR system as novel therapy for advanced cancers [11]. Although the anti VEGF-A monoclonal antibodies (MoAbs) proved beneficial effects, they still need to be associated with classical chemotherapy for optimal results [10]. They are thought to allow a better penetration of the cytotoxic agents into the tumor by temporarily normalizing the tumor vasculature [12].

Bevacizumab (Avastin ${ }^{\circledR}$ ) is a MoAb that blocks the VEGF-A, approved for human usage after showing statistically significant benefits in randomized clinical trials (RCTs) for the treatment of metastatic colorectal cancer (mCRC) in association with fluoropyrimidinebased chemotherapy regimens [13]. It was approved in first line setting after prolonging the mOS from 15.6 to 20.3 months and the median progression free survival (mPFS) from 6.2 to 10.6 months when added to IFL chemotherapy (irinotecan +5 fluorouracil (5-FU)) [14], also showing better mPFS when added to XELOX/Folfox 4 regimen (oxaliplatin +5 -FU/Capecitabine) [15]. It was later approved in 2 nd line setting after showing a benefit of 2.2 months in mOS, better mPFS and response rates (RR) [16]. It also gained approval for continuation usage in 2nd line after progression under first line treatment, in combination with a different chemotherapy protocol, after showing a benefit of 1.4 months in mOS versus 2 nd line chemotherapy without Bevacizumab [17]. It can also be administered as maintenance treatment, preferably in combination with a fluoropyrimidine derivative until progression or intolerable toxicity, with an advantage in PFS but not OS when compared with the "stop and go" strategy [18]. In the "adjuvant" setting (curative resection of metastasis) it did not confirm an advantage in terms of OS or PFS, so its administration remains controversial for the time being [19].

Other pathways involved in tumor development are activated consecutively to the interaction between the epidermal growth factor (EGF) and its receptor EGFR/
HER-1. The binding of the ligand to its receptor leads to the activation of the tyrosine kinase and of 2 downstream pathways (Ras-MAPK and PI-3k-Akt) that control cell proliferation, differentiation, migration, adhesion, inhibition of apoptosis, tumor invasion and metastatic spread. EGFR is overexpressed in many cancers such as CRC, head and neck (H\&N) and non-small cell lung cancers (NSCLC). Cetuximab (Erbitux ${ }^{\circledR}$ ) is a chimeric IgG1 MoAb that binds with a higher affinity than EGF to the EGFR, leading to an antitumor effect; it also has an antibody-dependent cell-mediated cytotoxic effect [20]. The KRAS and NRAS (all-ras) gene mutations lead to continuous activation of MAPK pathway despite the EGFR inhibition by Cetuximab [21]. BRAF gene mutations have been revealed in $\mathrm{mCRC}$ as independent negative predictive factors for lack of efficacy of anti EGFR antibodies in first line treatment of mCRC [22].

Cetuximab is currently approved for the treatment of all-ras wild type (wt), EGFR expressing mCRC, after having shown statistically significant results in RCTs [23]. Starting 2008 and 2013 respectively, the status of k-ras gene and all-ras afterwards were mandatory to be wt in order to administer the treatment [24]. It was first approved in combination with irinotecan in patients refractory to irinotecan or as monotherapy in recurrent disease with intolerance to irinotecan, after showing an improvement of 2.6 months in mPFS and improved RR compared to chemotherapy alone [25]. Later on, it gained approval as monotherapy after failure of irinotecan or oxaliplatin-based chemotherapy, with a 1.5 months benefit in mOS, better mPFS and RR; the benefits were shown to be present only in k-ras wt patients [26]. In 2012 it was also approved as first line treatment in combination with chemotherapy, after retrospective analyses of three randomized studies $[26,27,28,29]$.

The modified natural history of metastatic colorectal cancer by current treatments is similar to the one of a chronic disease that remains deadly. The improved survival of the patients permits the identification of the late adverse effects that can impair their quality of life.

\section{Cardiovascular (CV) adverse effects of Bevacizumab (HTN) \\ Bevacizumab-induced arterial hypertension}

It is the most frequent $\mathrm{CV}$ side effect of the anti VEGF drugs and it is more of an "on target" effect rather than an adverse one, as it mirrors the efficacy of VEGF inhibition; for this reason, prophylaxis is not possible. All potential mechanisms are related to VEGF inhibition: decreased NO production leading to vasoconstriction and increased peripheral vascular resistance; capillary rarefaction; endothelial dysfunctions with consecutive increase in endogenous secretion of endothelin 1; renal dysfunctions and others $[30,31,32,33]$. 
It is defined and graded according to the Common Terminology Criteria for Adverse Events (CTCAE) v.4 [34] as an increase in blood pressure (BP) at $>140 / 90 \mathrm{~mm}$ $\mathrm{Hg}$ or a symptomatic increase of $>20 \%$ of the diastolic BP. It is reversible and dependent on the duration of exposure and dose.

There seems to be a 3 fold increase in risk while on $2.5 \mathrm{mg} / \mathrm{kg} /$ week of Bevacizumab and a 7.5 fold increase in risk while on $5 \mathrm{mg} / \mathrm{kg} /$ week [35]. More than $90 \%$ of the patients treated will experience a BP elevation, out of which $22-24 \%$ develop HTN any grade and $8 \%$ grade $3-4$ $[33,36,37]$. It may occur at any time after the initiation of the treatment, most often being described after 1 month [30], or 3 months according to other authors (after 6 cycles) [38].

The population at high risk for developing Bevacizumab-induced HTN is represented by patients with previous HTN, age $>65$ years, $\mathrm{BMI} \geq 25$ [37], smoking, hypercholesterolemia [32].

Although no clear recommendation exists, it is well known that routine monitoring of the BP must be performed weekly during the first cycle, then 2-3 weeks and at least once before every administration [31]. For other anti VEGF drugs there is the rule of the 3 ambulatory measurements the average of 3 morning values at 5 minutes interval and the same in the evening, for 3 days/week [39].

Numerous studies have proved Bevacizumabinduced HTN to be predictive for treatment efficacy and better survival $[36,40]$, especially if onset is during the first 3 months [30,31].

The treatment has to be adapted to the patient; most commonly used are the Angiotensin-converting enzyme inhibitors (ACEi) or angiotensin receptor blockers (ARBs) and dihydropyridine calcium channel blockers (diltiazem, verapamil are not recommended due to CYP3A4 inhibition) [31,37]; according to some, nifedipine could induce VEGF synthesis [41]. A good control of HTN can reduce the number of heart failures.

\section{Arterial thromboembolic events (ATEs)}

In order of frequency, they mostly include myocardial, cerebrovascular or peripheral arterial events, probably due to the prevalence of the atherosclerotic plaques and the fact that the coronary arteries are more VEGF-dependent in maintaining their integrity [42]. Thus, the VEGF inhibition leads to arterial thrombosis $[43,44]$ and possibly impairs the development of collateral circulation [42].

The incidence of ATEs in RCTs varies from 1.7\% in chemo-treated patients to up to $3.8 \%$ in Bevacizumabcontaining regimens [13]. A meta-analysis [43] that included 13026 patients in 20 clinical trials showed that the addition of Bevacizumab to chemotherapy leads to a $46 \%$ increase in the risk of ATEs, mostly during the first 3 months. These conclusions are enforced by at least two other studies: a meta-analysis [42] on 12617 patients (6147 with
mCRC) from 20 RCTs, that showed that in Bevacizumabtreated patients the incidence of all grade ATEs was 3.3\% and of high-grade ATEs was $2.0 \%$, with a relative risk of 1.44 ( $p<0.013$ ); statistically significant higher levels were seen in mCRC and renal cell carcinoma patients; and the second study, a meta-analysis [45] on 4617 patients with solid tumors from 7 RCTs (chemo +/- Bevacizumab) that showed a summary incidence of Bevacizumab-induced cardiac ischemia of $1 \%$, with a significant relative risk of 2.49 , regardless of the dose administered (2.5 vs $5 \mathrm{mg} /$ $\mathrm{kg} /$ week); among the CRC patients, the relative risk was of 2.13. Regarding the 5-FU addition to Bevacizumab, there was no increased cardiovascular risk found with the association.

There are multiple risk factors for ischemic cardiac disease (ICD) in cancer patients: age, performance status, mobility, stage of the disease, histology, prothrombotic cancer states, chemotherapy [45] hypertension, diabetes, hyperlipemia, etc. [42]. Bevacizumab-induced ICD does not appear to be dependent on the duration of treatment $[43,46]$. Serious ATEs occur in $1 / 27$ treated patients [46].

Regarding the risk factors, the timing of ATEs and the use of aspirin prophylaxis in ATEs, a meta-analysis [44] including 1745 patients from 5 RCTs with metastatic tumors $(69 \% \mathrm{mCRC})$ that confirmed a significantly greater risk of ATEs associated with Bevacizumab use, also showed that the risk factors with statistical significance were a personal history of an ATE and age above 65. The median time to the ATE was of 2.1 months in the control group vs 2.6 in the Bevacizumab treated patients. The prophylactic aspirin ( $\leq 325 \mathrm{mg}$ /day) concomitant with Bevacizumab plus chemotherapy did not significantly increase the bleeding risk versus chemotherapy alone; patients with baseline risk factors for ATEs without aspirin prophylaxis had a higher incidence of ATEs then the ones that had it (22.9\% vs 3.4\%, $\mathrm{p}<0.03$ ).

\section{Venous thromboembolic events (VTEs)}

They include deep vein thrombosis (DVT), pulmonary embolism (PE) and thrombophlebitis. Cancer patients are already predisposed to VTEs due to advanced age, altered performance status, personal history of VTEs, tumor type, chemotherapy, surgery, etc. The involvement of Bevacizumab in VTEs is controversial. In clinical studies that used Bevacizumab, the incidence of VTEs varied between 2.8-17.3\% versus 3.2-15.6\% in patients without Bevacizumab, with no statistically significant difference between the 2 groups [13].

A meta-analysis [44] including 1745 patients from 5 RCTs with metastatic tumors that confirmed a significantly greater risk of ATEs associated with Bevacizumab use, did not show a significant risk of G3-G4 VTEs $(9.97 \%$ in Bevacizumab-treated vs $9.85 \%$ in the control group, $\mathrm{p}=0.44$ ).

A different meta-analysis [47] of 15 randomized studies that included 7956 patients, showed a summary 
incidence for any grade VTE of $11.9 \%$ and for G3-4 of $6.3 \%$ in Bevacizumab-treated patients, with a relative risk of 1.33 versus the chemo-treated patients $(p<0.001)$, regardless the dose of Bevacizumab. Hurwitz et al. [48] performed a metaanalysis on 6055 patients in 10 randomized trials (CRC, NSCLC, pancreatic cancer, breast cancer); there were no significant differences in the unadjusted incidence of VTE (patients with Bevacizumab had longer follow-up due to longer treatment because of good responses) between the Bevacizumab vs control $(10.9 \%$ vs $9.8 \%, \mathrm{p}<0.13)$. In the same study, the use of therapeutic oral anticoagulation for VTEs did not lead to more severe bleedings in the Bevacizumab group.

\section{Heart failure (HF)}

The risk of Bevacizumab-induced HF was predominantly described in RCTs on breast cancer and lymphoma, in patients that had concurrent or previous anthracycline treatment or a personal history of mediastinal radiotherapy [13] and for some, in the context of previous Trastuzumab therapy.

The global incidence of congestive HF in a metaanalysis on breast cancer [49] that included 5 RCTs on 3784 patients, was of $1.6 \%$ in patients treated with Bevacizumab and $0.4 \%$ in the chemo-treated without Bevacizumab patients, with a significant relative risk of 4.74 . One of the limitations of the study is that it could only analyze the results of the individual clinical trials and not the individual patient data, so there is no information available neither regarding previous exposure to chemotherapy (especially anthracyclines well-known as cardiotoxic drug), nor regarding patients' comorbidities. It is known that other anti VEGF molecules can induce a reversible cardiotoxicity and sometimes treatment can be resumed with lower doses after complete remission of the HF - although at this point, little is known about Bevacizumab in this regard [50].

The mechanism of Bevacizumab-induced cardiotoxicity is not clear. Blocking the VEGF can interfere with the repair of myocardial damage and impair the collateral vessel formation. Bevacizumab-induced HTN leads to cardiomyocitic ischemia, secretion of HIF-1 and consecutive increase in angiogenic factors; blocking VEGF in these conditions aggravates the situation [51].

RCTs in Bevacizumab registration trials in mCRC did not report any Bevacizumab-induced HF $[14,15,16,52,53]$. In a retrospective analysis performed on 6937 patients with mCRC enrolled in 15 RCTs [54], there was no association found between Bevacizumab treatment and congestive HF or cardiac death. Another retrospective study [55] on 6803 elderly patients with $\mathrm{mCRC} \geq 65$ years of age also showed no connection between Bevacizumab treatment and congestive HF/cardiomyopathy or cardiac death. Prospective studies with this primary endpoint are needed in order to conclude on this issue. The treatment of this adverse event consists of ACEi, ARBs and betablockers.

\section{Cardiovascular (CV) side effects of Cetuximab}

$\mathrm{RCTs}$ that lead to Cetuximab registration in $\mathrm{mCRC}$ did not report at the time any direct cardiac adverse events. In H\&N cancers where it is usually administered with both 5-FU and Cisplatin or concurrent with radiotherapy, there was a higher incidence of cardiorespiratory arrests and sudden cardiac deaths reported. Being a chimeric MoAb (murine + human) its administration is engraved with infusion-related allergic events that can be severe in $2-5 \%$ of the patients and fatal in $<1 / 1000$ patients; they usually appear during the first hour of the first administration but can also appear after a few hours or during subsequent infusions, regardless of antihistaminic premedication. Patients can experience bronchospasm, urticaria and cardiovascular symptoms as arterial hypotension, and in more rare cases angina, myocardial infarction, cardiac failure, shock, sudden death [56].

\section{Venous thromboembolic events (VTES)}

There is limited information regarding Cetuximabinduced VTEs or ATEs in mCRC patients; these were not reported as adverse events in the registration trials. Taking into account the post marketing experience as well, the official product information [23] includes as less frequent side effects, the DVT and PE $(\geq 1 / 1000$ and $<1 / 100$ cases respectively).

One of the few studies performed in this regard, a meta-analysis of RCTs published in 2012 [57] evaluated the incidence and relative risk of VTEs and ATEs in patients treated with antiEGFR drugs, either MoAbs or tyrosinekinase inhibitors (TKIs) in patients with different advanced cancers. Regarding the VTEs, there were 7073 patients included in the analyses from 11 phase 2-3 randomized trials in $\mathrm{H} \& \mathrm{~N}, \mathrm{NSCLC}$ and $\mathrm{CRC}$; the incidence was of $5 \%$ for patients with antiEGFR treatments vs $3.7 \%$ in the control group, with statistically significant difference between the MoAbs and TKI's (5.9 vs $2.6 \%, \mathrm{p}<0.001$ ); the relative risk of VTEs was also higher for patients treated with antiEGFR drugs, statistically significant for the MoAbs. The difference between the MoAbs and TKIs regarding their potential to induce VTE is probably explained by the concurrent chemotherapy administered with the MoAbs, whose thromboembolic potential is well known. Regarding the ATE, there was no significant difference between the experimental vs control arms.

One of the mechanisms that can explain the occurrence of VTEs resides in the antiangiogenic activity of Cetuximab. At the same time, the concurrent chemotherapy could also be an important factor. It is known that Cisplatin, used in combination with Cetuximab in $\mathrm{H} \& \mathrm{~N}$ cancers has an important thromboembolic risk and also, fluoropyrimidines used in both $\mathrm{H} \& \mathrm{~N}$ and $\mathrm{CRC}$ can induce cardiac adverse events [57]. 


\section{Defects in left ventricular depolarization and repolarization}

There are certain oncological treatments, chemotherapy and molecular treatments that are known to have a risk of prolonging the QT interval on electrocardiogram (ECG). The QT interval represents the total duration of cardiac depolarization and repolarization; its prolongation signifies a late repolarization that can lead to ventricular arrhythmias including "torsade des points" that can rapidly progress to ventricular fibrillation and sudden death [58]. QT interval value needs correction (QTc) according to heart rate and there are different formulas used in practice such as Bazett, Framingham, Fridericia. Long QT can also be induced by left ventricular hypertrophy, cardiac ischemia, dyselectrolytemia, psychotropic drugs, antimicrobials, antihistamines, etc [59].

There have been some observations that Cetuximab administration is sometimes related to QTc prolongation. Through the adverse event reporting, the clinical trials did not manage to establish a relationship between the two. The only prospective clinical study [60] identified in the literature with this primary objective was performed in 20 centers in USA, on 51 patients treated with Cetuximab for advanced H\&N, NSCLC, CRC and other cancers, treated with Cetuximab monotherapy. Serial ECG monitoring was performed and also dosing of seric Cetuximab concentration; from all patients, 37 were included in the final analyses. The study concluded that monotherapy with Cetuximab did not have clinically significant effects on QTc interval, PR interval, QRS interval or on cardiac frequency.

Dyselectrolytemia is an important cause of QTc prolongation and especially hypomagnesaemia. Cetuximab is known to induce it very frequently $(>1 / 10$ patients treated). This can be one mechanism through which Cetuximab can induce prolonged QTc, although based on the currently available data, a direct mechanism cannot be excluded and this warrants further prospective studies with a higher number of patients to be included.

\section{Cardiac evaluation in patients receiving Bevacizumab and Cetuximab}

There are no clear recommendations regarding the type, timing and frequency of investigations in order to prevent or early detect a CV adverse effect of these drugs, as they exist for anthracyclines or Trastuzumab, for example. They all need to be individualized according to patient's needs, preexisting cardiac or non-cardiac comorbidities, previous treatments, type of cancer and choice of concurrent oncological treatment. All the existing recommendations are based on expert opinion and are not evidence-based. Risk scores would be very helpful in early identification of patients at risk, but the ones that exist were not validated in prospective clinical trials. It is mandatory that all patients have a thorough anamnesis and clinical evaluation at baseline, in order to identify all preexisting conditions and baseline risk factors for a cardiovascular disease. In the BEAT study [61] that had as primary endpoint the safety of Bevacizumab plus first-line chemotherapy in 1914 mCRC patients, the on-study assessments consisted of the evaluation of medical history, physical examination, blood and urine works; more specific cardiac work-up was not mandatory and at physician's discretion. But we have to take into account that the patients included in clinical trials are well selected, with a good performance status and without clinically significant $\mathrm{CV}$ comorbidities.

In 2016, the European Society of Cardiology published a position paper on cancer treatments and CV toxicity with general recommendations. Regarding a possible treatment-induced myocardial dysfunction or HF there are many possible options for diagnosis; it is important to follow the same method in a patient's surveillance. ECG is recommended in all these patients at baseline, during the treatment and follow-up. The imaging methods that can be used are represented by echocardiography (preferred), nuclear cardiac imaging (MUGA), cardiac magnetic resonance imaging (MRI). Cardiac biomarkers (troponin I), natriuretic peptides (brain natriuretic peptide (BNP), NTproBNP), can detect even subclinical damages, but there is no evidence-based practice when an abnormal value is met regarding the interruption or permanent discontinuation of the drug [62].

\section{Novel biomarkers for cardiovascular toxicities}

The progress made in the field of research and genetics made it possible to search for new biomarkers. In a pooled analysis on 1631 patients treated with Bevacizumab for advanced cancers, with available DNA for testing, genetic variations in VEGF-A pathway or other genes involved in HTN (ten single nucleotide polymorphisms) were positively associated with Bevacizumab-induced HTN [63].

MicroRNAs (miRs) are small, single stranded RNA molecules of 18-25 nucleotides that do not encode for protein synthesis, but have a role in regulating gene expression at a posttranscriptional level [64]. A prospective study [65] was conducted in order to establish the implication of miRs in Bevacizumab-induced cardiotoxicity; they included 88 consecutive patients with Bevacizumab-induced, confirmed cardiac HF within 24 hours from the onset, 90 colorectal cancer control volunteers with Bevacizumab treatment and 66 patients with acute myocardial infarction without Bevacizumab treatment. Two circulating miRs (out of 19) were found to be specifically elevated in patients with Bevacizumab-induced cardiotoxicity: miR1254 and miR579. Other prospective studies are needed, but these results raise enthusiasm not only regarding early and morespecific diagnosis, but also regarding new therapeutic targets. 


\section{Acknowledgement}

Genetic polymorphism and its predictive role in chemotherapy of the metastatic colorectal cancer project acronym GEMIRCORE. Project code: PN-II-RUTE-2014-4-2830.

\section{References}

1. Labianca R, Nordlinger B, Beretta GD, Mosconi S, Mandala M, Cervantes A, et al. Early colon cancer: ESMO Clinical Practice Guidelines for diagnosis, treatment and follow-up. Ann Oncol. 2013;24 suppl 6:vi64-vi72.

2. Van Cutsem E, Cervantes A, Adam R, Sobrero A, Van Krieken JH, Aderka D, et al. ESMO consensus guidelines for the management of patients with metastatic colorectal cancer. Ann Oncol. 2016;27(8):1386-1422.

3. Tournigand C, Andre T, Achille E, Lledo G, Flesh M, MeryMignard D, et al. FOLFIRI followed by FOLFOX6 or the reverse sequence in advanced colorectal cancer: a randomized GERCOR study. J Clin Oncol. 2004;22(2):229-237.

4. Heinemann V, von Weikersthal LF, Decker T, Kiani A, VehlingKaiser U, Al-Batran SE, et al. FOLFIRI plus cetuximab versus FOLFIRI plus bevacizumab as first-line treatment for patients with metastatic colorectal cancer (FIRE-3): a randomised, openlabel, phase 3 trial. Lancet Oncol. 2014; 15(10):1065-1075.

5. Ferlay J, Steliarova-Foucher E, Lortet-Tieulent J, Rosso S, Coebergh JW, Comber H, et al. Cancer incidence and mortality patterns in Europe: estimates for 40 countries in 2012. Eur J Cancer. 2013;49(6):1374-1403.

6. Hanahan D, Coussens LM. Accessories to the crime: functions of cells recruited to the tumor microenvironment. Cancer Cell. 2012;21(3):309-322.

7. Folkman J. Tumor angiogenesis: therapeutic implications. N Engl J Med. 1971;285(21):1182-1186.

8. Hoeben A, Landuyt B, Highley MS, Wildiers H, Van Oosterom AT, De Bruijn EA. Vascular endothelial growth factor and angiogenesis. Pharmacol Rev. 2004;56(4):549-580.

9. Ferrara N. VEGF and the quest for tumour angiogenesis factors. Nat Rev Cancer. 2002;2(10):795-803.

10. Kerbel RS, Ellis LM. Angiogenesis. In: DeVita VT, Lawrence TS, Rosenberg SA, DePinho RA, Weinberg RA (eds) DeVita, Hellman, and Rosenberg's Cancer Principles and Practice of Oncology. 9th ed. Philadelphia: Lippincott Williams \&Wilkins; 2011, pp 101-112.

11. Shibuya M. Vascular Endothelial Growth Factor (VEGF) and Its Receptor (VEGFR) Signaling in Angiogenesis: A Crucial Target for Anti- and Pro-Angiogenic Therapies. Genes Cancer. 2011;2(12):1097-1105.

12. Jain RK. Normalization of tumor vasculature: an emerging concept in antiangiogenic therapy. Science. 2005;307(5706):5862.

13. European Medicines Agency. Avastin Summary of Product Characteristics. Available from: http://www.ema.europa.eu/ docs/en_GB/document_library/EPAR_-_Product_Information/ human/000558/WC500029119.pdf

14. Hurwitz H, Fehrenbacher L, Novotny W, Cartwright T, Hainsworth J, Heim W, et al. Bevacizumab plus irinotecan, fluorouracil, and leucovorin for metastatic colorectal cancer. N Engl J Med. 2004;350(23):2335-2342.

15. Saltz LB, Clarke S, Díaz-Rubio E, Scheithauer W, Figer A, Wong R, et al. Bevacizumab in combination with oxaliplatin-based chemotherapy as first-line therapy in metastatic colorectal cancer: a randomized phase III study. J Clin Oncol. 2008;26(12):20132019.

16. Giantonio BJ, Catalano PJ, Meropol NJ, O'Dwyer PJ, Mitchell EP, Alberts SR, et al. Bevacizumab in combination with oxaliplatin, fluorouracil, and leucovorin (FOLFOX4) for previously treated metastatic colorectal cancer: results from the Eastern Cooperative Oncology Group Study E3200. J Clin Oncol 2007;25(12):1539-1544.

17. Bennouna J, Sastre J, Arnold D, Österlund P, Greil R, Van Cutsem E, et al; ML18147 Study Investigators. Continuation of bevacizumab after first progression in metastatic colorectal cancer (ML18147): a randomised phase 3 trial. Lancet Oncol. 2013;14(1):29-37.

18. Zhao L, Wang J, Li H, Che J, Cao B. Meta-analysis comparing maintenance strategies with continuous therapy and complete chemotherapy-free interval strategies in the treatment of metastatic colorectal cancer. Oncotarget. 2016;7(22):33418-33428.

19. Turan N, Benekli M, Dane F, Unal OU, Kara HV, Koca D, et al. Adjuvant systemic chemotherapy with or without bevacizumab in patients with resected pulmonary metastases from colorectal cancer. Thorac Cancer. 2014;5(5):398-404.

20. Lenz HJ. Cetuximab in the management of colorectal cancer. Biologics. 2007;1(2):77-91

21. Allegra CJ, Rumble RB, Hamilton SR, Mangu PB, Roach $\mathrm{N}$, Hantel A, et al. Extended RAS Gene Mutation Testing in Metastatic Colorectal Carcinoma to Predict Response to AntiEpidermal Growth Factor Receptor Monoclonal Antibody Therapy: American Society of Clinical Oncology Provisional Clinical Opinion Update 2015. J Clin Oncol. 2016;34(2):179-185. 22. Gong J, Cho M, Fakih M. RAS and BRAF in metastatic colorectal cancer management. J Gastrointest Oncol. 2016;7(5):687-704

23. European Medicines Agency. Summary of Product Characteristics Erbitux. Available from: http://www.ema. europa.eu/docs/ro_RO/document_library/EPAR__Product_ Information/human/000558/WC500029119.pdf

24. US Food \& Drug Administration. FDA Approves Cetuximab (Erbitux) and Panitumumab (Vectibix). Available from:http://www.fda.gov/AboutFDA/CentersOffices/ OfficeofMedicalProductsandTobacco/CDER/ucm172905.htm

25. Cunningham D, Humblet Y, Siena S, Khayat D, Bleiberg H, Santoro A, et al. Cetuximab monotherapy and cetuximab plus irinotecan in irinotecan-refractory metastatic colorectal cancer. N Engl J Med. 2004;351(4):337-345.

26. Jonker DJ, O'Callaghan CJ, Karapetis CS, Zalcberg JR, Tu D, $\mathrm{Au}$ HJ, et al. Cetuximab for the treatment of colorectal cancer. N Engl J Med. 2007;357(20):2040-2048.

27. Van Cutsem E, Köhne C, Hitre E, Zaluski J, Chang Chien CR, Makhson A, et al. Cetuximab and chemotherapy as initial treatment for metastatic colorectal cancer. $\mathrm{N}$ Engl J Med. 2009;360(14):1408-1417.

28. Bokemeyer C, Bondarenko I, Makhson A, Hartmann JT, Aparicio J, de Braud F, et al. Fluorouracil, leucovorin, and oxaliplatin with and without cetuximab in the first-line treatment of metastatic colorectal cancer. J Clin Oncol. 2009;27(5):663-671. 29. Bokemeyer C, Köhne CH, Ciardiello F, Lenz HJ, Heinemann V, Klinkhardt U, et al. FOLFOX4 plus cetuximab treatment and RAS mutations in colorectal cancer. Eur J Cancer. 2015;51(10):12431252.

30. Österlund P, Soveri LM, Isoniemi H, Poussa T, Alanko 
T, Bono P. Hypertension and overall survival in metastatic colorectal cancer patients treated with bevacizumab-containing chemotherapy. Br J Cancer. 2011;104(4):599-604.

31. Brinda BJ, Viganego F, Vo T, Dolan D, Fradley MG. AntiVEGF-Induced Hypertension: a Review of Pathophysiology and Treatment Options. Curr Treat Options Cardiovasc Med. 2016 May;18(5):33.

32. Small HY, Montezano AC, Rios FJ, Savoia C, Touyz RM. Hypertension due to antiangiogenic cancer therapy with vascular endothelial growth factor inhibitors: understanding and managing a new syndrome. Can J Cardiol. 2014;30(5):534-543.

33. Ranpura V, Pulipati B, Chu D, Zhu X, Wu S. Increased risk of high-grade hypertension with bevacizumab in cancer patients: a meta-analysis. Am J Hypertens. 2010;23(5):460-468.

34. U.S. Department of Health and Human Services. Common Terminology Criteria for Adverse Events v4.0. Published: May 28, 2009 (v4.03: June 14, 2010). Available from: https:// evs.nci.nih.gov/ftp1/CTCAE/CTCAE_4.03_2010-06-14_ QuickReference_5x7.pdf

35. Zhu X, Wu S, Dahut WL, Parikh CR. Risks of proteinuria and hypertension with bevacizumab, an antibody against vascular endothelial growth factor: systematic review and meta-analysis. Am J Kidney Dis. 2007;49(2):186-193.

36. Cai J, Ma H, Huang F, Zhu D, Bi J, Ke Y, et al. Correlation of bevacizumab-induced hypertension and outcomes of metastatic colorectal cancer patients treated with bevacizumab: a systematic review and meta-analysis. World J Surg Oncol. 2013 Nov 28;11:306.

37. Wasserstrum Y, Kornowski R, Raanani P, Leader A, Pasvolsky $\mathrm{O}$, Iakobishvili Z. Hypertension in cancer patients treated with anti-angiogenic based regimens. Cardio-Oncology. 2015;1:6.

38. Nakaya A, Kurata T, Yokoi T, Iwamoto S, Torii Y, Katashiba Y, et al. Retrospective analysis of bevacizumab-induced hypertension and clinical outcome in patients with colorectal cancer and lung cancer. Cancer Med. 2016;5(7):1381-1387.

39. Wu S, Chen JJ, Kudelka A, Lu J, Zhu X. Incidence and risk of hypertension with sorafenib in patients with cancer: a systematic review and meta-analysis. Lancet Oncol. 2008;9:117-123.

40. Scartozzi M, Galizia E, Chiorrini S, Giampieri R, Berardi R, Pierantoni C, et al. Arterial hypertension correlates with clinical outcome in colorectal cancer patients treated with first-line bevacizumab. Ann Oncol. 2009;20(2):227-230.

41. Izzedine H, Ederhy S, Goldwasser F, Soria JC, Milano G, Cohen A, et al. Management of hypertension in angiogenesis inhibitor-treated patients. Ann Oncol. 2009;20(5):807-815.

42. Ranpura V, Hapani S, Chuang J, Wu S. Risk of cardiac ischemia and arterial thromboembolic events with the angiogenesis inhibitor bevacizumab in cancer patients: a meta-analysis of randomized controlled trials. Acta Oncol. 2010;49(3):287-297.

43. Schutz FA, Je Y, Azzi GR, Nguyen PL, Choueiri TK. Bevacizumab increases the risk of arterial ischemia: a large study in cancer patients with a focus on different subgroup outcomes. Ann Oncol. 2011;22(6):1404-1412.

44. Scappaticci FA, Skillings JR, Holden SN, Gerber HP, Miller $\mathrm{K}$, Kabbinavar F, et al. Arterial thromboembolic events in patients with metastatic carcinoma treated with chemotherapy and bevacizumab. J Natl Cancer Inst. 2007;99(16):1232-1239.

45. Chen XL, Lei YH, Liu CF, Yang QF, Zuo PY, Liu CY, et al. Angiogenesis inhibitor bevacizumab increases the risk of ischemic heart disease associated with chemotherapy: a metaanalysis. PLoS One. 2013 Jun 20; 8(6):e66721. doi: 10.1371/ journal.pone.0066721.

46. Tebbutt NC, Murphy F, Zannino D, Wilson K, Cummins MM, Abdi E, et al. Risk of arterial thromboembolic events in patients with advanced colorectal cancer receiving bevacizumab. Ann Oncol. 2011;22(8):1834-1838.

47. Nalluri SR, Chu D, Keresztes R, Zhu X, Wu S. Risk of venous thromboembolism with the angiogenesis inhibitor bevacizumab in cancer patients: a meta-analysis. JAMA. 2008;300(19):22772285 .

48. Hurwitz HI, Saltz LB, Van Cutsem E, Cassidy J, Wiedemann J, Sirzén F, et al. Venous thromboembolic events with chemotherapy plus bevacizumab: A pooled analysis of patients in randomized phase II and III studies. J Clin Oncol. 2011;29(13):1757-1764.

49. Choueiri TK, Mayer EL, Je Y, Rosenberg JE, Nguyen PL, Azzi GR, et al. Congestive heart failure risk in patients with breast cancer treated with bevacizumab. J Clin Oncol. 2011;29(6):632638.

50. Schmidinger M, Zielinski CC, Vogl UM, Bojic A, Bojic M, Schukro C, et al. Cardiac toxicity of sunitinib and sorafenib in patients with metastatic renal cell carcinoma. J Clin Oncol. 2008;26(32):5204-5212.

51. Verma N, Swain SM. Bevacizumab and heart failure risk in patients with breast cancer: a thorn in the side? J Clin Oncol. 2011;29(6):603-606.

52. Kabbinavar FF, Schulz J, McCleod M, Patel T, Hamm JT, Hecht JR, et al. Addition of bevacizumab to bolus fluorouracil and leucovorin in first-line metastatic colorectal cancer: results of a randomized phase II trial. J Clin Oncol. 2005;23(16):3697-3705. 53. Kabbinavar F, Hurwitz HI, Fehrenbacher L, Meropol NJ, Novotny WF, Lieberman G, et al. Phase II, randomized trial comparing bevacizumab plus fluorouracil (FU)/leucovorin (LV) with FU/LV alone in patients with metastatic colorectal cancer. J Clin Oncol. 2003;21(1):60-65.

54. Dai F, Shu L, Bian Y, Wang Z, Yang Z, Chu W, et al. Safety of bevacizumab in treating metastatic colorectal cancer: a systematic review and meta-analysis of all randomized clinical trials. Clin Drug Investig. 2013;33(11):779-788.

55. Tsai HT, Marshall JL, Weiss SR, Huang CY, Warren JL, Freedman AN, et al. Bevacizumab use and risk of cardiovascular adverse events among elderly patients with colorectal cancer receiving chemotherapy: a population-based study. Ann Oncol. 2013;24(6):1574-1579.

56. Drugs.com. Erbitux Side Effects in Detail - Drugs.com. Available from: https://www.drugs.com/sfx/erbitux-sideeffects.html

57. Petrelli F, Cabiddu M, Borgonovo K, Barni S. Risk of venous and arterial thromboembolic events associated with anti-EGFR agents: a meta-analysis of randomized clinical trials. Ann Oncol. 2012;23(7):1672-1679.

58. van Noord C, Eijgelsheim M, Stricker BH. Drug- and non-drug-associated QT interval prolongation. Br J Clin Pharmacol. 2010;70(1):16-23.

59. Strevel EL, Ing DJ, Siu LL. Molecularly targeted oncology therapeutics and prolongation of the QT interval. J Clin Oncol. 2007;25(22):3362-3371.

60. Deeken JF, Shimkus B, Liem A, Hill D, Gurtler J, Berghorn $\mathrm{E}$, et al. Evaluation of the relationship between cetuximab therapy and corrected QT interval changes in patients with advanced malignancies from solid tumors. Cancer Chemother Pharmacol. 2013;71(6):1473-1483. 
61. Van Cutsem E, Rivera F, Berry S, Kretzschmar A, Michael M, DiBartolomeo M, et al. Safety and efficacy of firstline bevacizumab with FOLFOX, XELOX, FOLFIRI and fluoropyrimidines in metastatic colorectal cancer: the BEAT study. Ann Oncol. 2009;20(11):1842-1847.

62. Zamorano JL, Lancellotti P, Rodriguez Muñoz D, Aboyans V, Asteggiano R, Galderisi M, et al. 2016 ESC Position Paper on cancer treatments and cardiovascular toxicity developed under the auspices of the ESC Committee for Practice Guidelines: The Task Force for cancer treatments and cardiovascular toxicity of the European Society of Cardiology (ESC). Eur Heart J.
2016;37(36):2768-2801.

63. Lambrechts D, Moisse M, Delmar P, Miles DW, Leighl N, Escudier B, et al. Genetic markers of bevacizumab-induced hypertension. Angiogenesis. 2014;17(3):685-694.

64. Lièvre A. Implications et perspectives d'application clinique des microARN en cancerologie: exemple du cancer colorectal. Cancéro dig. 2011;3(3):194-199.

65. Zhao Z, He J, Zhang J, Liu M, Yang S, Li N, et al. Dysregulated miR1254 and miR579 for cardiotoxicity in patients treated with bevacizumab in colorectal cancer. Tumor Biol. 2014;35(6):52275235 . 